\title{
Performance-Based Design Approach for Tailored Acoustic Surfaces
}

\author{
Andrea Giglio, Ingrid Paoletti and Maia Zheliazkova
}

\begin{abstract}
The acoustics of architecture has a large influence on the physical and psychological state of people, impacting on their communication, concentration and behaviour. Nevertheless, the sound issues of the interior of common spaces are often faced in the last stage of the design process, as an accurate acoustic design requires a high level of expertise. Owners, companies, public administration, and so on face this issue by selecting off-the-shelf acoustic panels or furniture, which might not always respond to the acoustic requirements, specific for each space. This leads to unsatisfying acoustic conditions, consequently increasing the initial investment. Nowadays, a new awareness on the topic of architectural acoustics design has pushed towards finding new integrated methodologies able to deliver tailored solutions for the design with sound, which embed performance criteria early in the design phase by means of simulation and computational techniques. This paper describes a workflow developed in the frame of the research project: "EcoAcustica. Sustainable and innovative surfaces for adaptive acoustics", aimed at creating acoustic surfaces and customised to improve the interior acoustics in a global geometry scale. The design of the new ABC Department Digital Fabrication Lab at Politecnico di Milano is used as a case study for the proposed methodology.
\end{abstract}

Keywords Design stage $\cdot$ Simulation $\cdot$ Applied research $\cdot$ Architectural acoustics $\cdot$ Performance-based design

\section{Introduction}

EcoAcustica project emerges from the collaboration between the innovative composite materials system, developed by Woodskin ${ }^{\circledR}$ and TecnoSugheri, the main importer

\footnotetext{
A. Giglio ( $\varangle)$ · I. Paoletti · M. Zheliazkova

Architecture, Built Environment and Construction Engineering-ABC Department, Politecnico di Milano, Milan, Italy

e-mail: andrea.giglio@polimi.it 
and manufacturer of the eco-sustainable thermoacoustic CORKPAN panels, supported by the scientific expertise of ABC Department at Politecnico di Milano in advanced technological research for architecture.

Implementing innovative computational design and fabrication methods, the project develops a customisable system aimed at providing high-performance acoustics for interiors and tailored according to their functional and spatial characteristics. The control of the acoustic conditions is achieved at two levels: a global level of the overall geometry for controlling early reflections and sound scattering, and on local material level through sandwich composites differentiation and surface treatment for tuning sound absorption.

The origami-like structure of the Woodskin ${ }^{\circledR}$ technology allows in achieving this degree of adaptivity/flexibility. The system combines flexible textile sandwiched between two layers of solid material (mainly wood). Once glued together, in the solid flat surfaces, cuts are created that allow them to be flexed away from the rigid form, thus creating a desired three-dimensional shape.

EcoAcustica project fosters the contamination between academically led research and the craftsmanship of small and medium enterprises (SMEs), and works towards the introduction of innovation and excellence in the supply chain within the Industry 4.0 research agenda. This effective collaboration enables the synergy between the two SMEs involved, creating an environment for applying in an industrial context an innovative process for design and fabrication of fully customisable acoustic panels.

\section{Room Acoustics Modelling Techniques}

The quality of acoustics in an enclosed space is affected by the geometrical and material properties of its surfaces (Cox and D'Antonio 2017). When the sound wave strikes a surface it can be absorbed, transmitted or reflected. The achievement of high-comfort conditions for humans depends on the control of these phenomena combined with considerations on the functional programme of the space.

Every functional programme has highly specific sound requirements: the sound conditions for speech are not the same as those for music events and vice versa. The main value used universally to guarantee proper sound conditions is the reverberation time $\left(\mathrm{T}_{60}\right)$, defined as the time required for the sound in an enclosed space to decay by $60 \mathrm{~dB}$ (ISO 3382-1). This in turn defines the amount of absorptive surfaces needed in the room. For example, for proper speech intelligibility, the reverberation time required is of about $1 \mathrm{~s}$, while for symphonic music it is $2 \mathrm{~s}$.

\subsection{Simulating Acoustic Phenomena}

Various room acoustics modelling techniques and computer models have been developed over the last few decades (Rindel 1995). Nowadays, highly accurate predictions 
are achievable, thanks to custom software that can be more easily adapted to the designers' needs than the classic standalone ones.

The most important room acoustic techniques are: ray tracing, image-based methods, combined methods and beam tracing.

The ray tracing method creates a dense spread of rays, which are reflected around a room and tested for intersection with a spherical detector (Kulowski 1984; Lehnert 1993). This one is in part passed by the beam tracing method that considers ray with volume using conical (Van Maerck and Martin 1993) or triangular beams (Lewers 1993). The image method considers the rays calculating images of the sound sources in reflecting walls (Lee and Lee 1988). In this way, it can provide more accurate information at a point detector (Allen and Berkley 1979). The most used method combines the potentialities of ray tracing and image methods to get room acoustical impulse responses (Vorlander 1989).

Through the application of the finite element method (FEM), the original problem of determining field variable distributions in a continuum domain is approximately transformed into a problem of determining the field variables at some discrete (nodal) positions within each element (Gladwell 1965). The method is usually used for propagation of sound vibration in the structures (Jiang et al. 2011).

The boundary element method (BEM) is derived through the discretisation of an integral equation that is mathematically equivalent to the original partial differential equation. It is used mainly in applications such as the sound output of a loudspeaker, the noise from a radiating source such as an engine and the interior acoustic modes of an enclosure (Hargreaves and Cox 2010).

\subsection{Integrated Approaches for Architectural Acoustics}

Although the room acoustics modelling techniques have originally been developed for the acoustic prediction and design of spaces for music (theatres, concert halls, auditoria etc.), the problems are equally challenging in other typologies of rooms and, thanks to the computational processes, the same methods can be adopted for them.

The main problem is that the reverberation time cannot be calculated only by the classical equations of Sabine and Eyring because the rooms can be very irregular, the diffusion of sound can be uneven and very different from the simple assumption of a diffuse sound field, and the sound absorption can be distributed irregularly over the surfaces. Another problem is that the reverberation time is not the most complete parameter describing the complex acoustical conditions of a room. Parameters, such as speech transmission index (STI) for workrooms and open plan offices, sound pressure level $(\mathrm{SPL})$, clarity $\left(\mathrm{C}_{50}\right)$ or definition $\left(\mathrm{D}_{50}\right)$ for auditoria, should also be considered in the acoustic design of those spaces.

Nowadays, 3D computer modelling and numerically controlled fabrication processes have introduced new territories for the design of architectural acoustics. With 3D modelling programmes such as Rhino3D and Grasshopper incorporating 
NURBS, geometries can be tailored to meet aesthetic, functional and performance objectives. These processes also allow embedding acoustic simulation in the initial design stages. Computational and optimisation design tools are taking a stronger role in geometrical acoustics. Mathematically defined geometries can be manipulated and exchanged between the modelling and analysis programmes, allowing for an easier shaping of rooms and surfaces (Bassuet et al. 2014).

Computational design processes applied to architectural acoustics help:

- To balance the relation between the shape of the space and sound comfort;

- To assist designers' creativity and explore a wide range of options but at the same time narrowing the field of possible solutions to only the most fitted ones;

- To check and confirm the validity of a particular design, and potentially aim towards more deterministic solutions, reducing the risk of wasting time or material;

- To define a novel performance-driven aesthetics;

- To develop techniques coupling in an auditory model a set of subjective criteria or spatial parameters, used for design optimisation.

\section{Methodology}

\subsection{Performance-Driven Design for Global Form-Finding of Acoustic Surfaces}

In response to these points, the paper describes an approach to the generation of acoustic surfaces with tailored performance for absorption and diffusion, employing simulation and evaluation tools. FEM analysis of the sound pressure level in the time and frequency domains is used for the identification of potentially problematic acoustic zones and frequency ranges, and accurate placement of acoustically absorptive surfaces, followed by ray-tracing as a means for formal definition of these surfaces on the global scale of the panel. These two performance criteria are used as the main design generation drivers of two geometric configurations: a ceiling and a wall partition, and are implemented in a parametric 3D design environment-Grasshopper for Rhino3D. Focusing on maximising the absorption by means of orienting the analysed geometries such as they intersect as many rays as possible, while minimising the overall surface area, here multi-objective optimisation algorithms are used for the generation of possible options. The goal is applying this performance-based strategy for the design of acoustic surfaces and solutions that are tailored not only to fit a specific acoustic condition requirement but also to minimise production cost and waste.

The objective of the EcoAcustica project towards an efficient manufacturing process is addressed by using the flexible Wood-Skin technological system for the generation of developable surfaces with planar triangulated facets. Such geometries allow to be produced from a single flat sheet by numerically controlled milling procedures, 

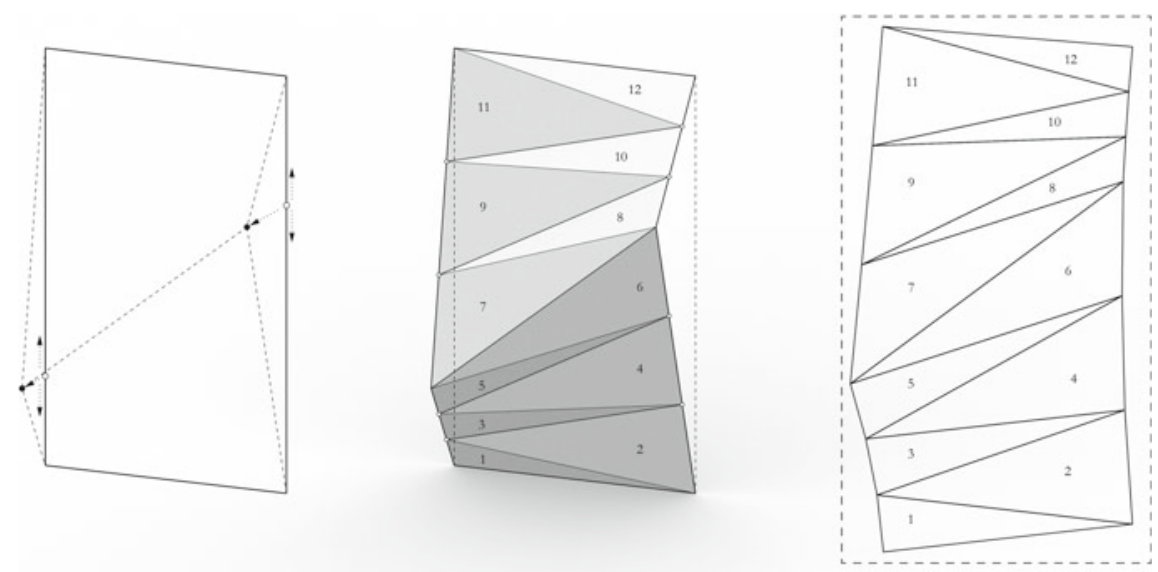

Fig. 1 Design logic of the developable surfaces

which guarantees reduced material waste and a facilitated assembly process that eliminates complex joinery (Fig. 1).

\subsection{Empirical Studies on Materials and Absorption Coefficients}

The integration of the ICB cork as the absorptive component of the acoustic system plays a central role in the EcoAcustica project. Four main types of new base composites were designed, so as to allow the differentiation of acoustic properties. Each composite targets a specific behaviour (absorption, diffusion and combination of absorption and diffusion), combining an acoustically reflective material, like okoume or plywood, with absorptive one-ICB cork, the most effective in mid- to high-frequency range. Following the fabrication logic of the Wood-Skin system, the combination of various composites within the same element allows for the versatility of the overall system. The absorptive properties of the panels are also enhanced with the possibility of varying the air gap behind that the Wood-Skin system allows. Based on preliminary studies of the behaviour of these material composites, prototype panels have been developed and tested according to the ISO 354:2003 standard. These empirical data on the sandwich composition and absorption coefficients are used in the simulation phase. 


\section{Case Study}

\subsection{Case Study Selection and Criteria}

This paper applies the proposed methodology for the acoustics optimisation of the new laboratory of Department ABC at Polytechnic of Milan. The space, originally used as a classroom, has been designed to accommodate a new lab for advanced digital fabrication and prototyping for architecture. It hosts the following functions:

- Two fabrication areas with numerically controlled machinery-an area for robotic fabrication and a space for FDM 3D printers and a CNC machine (Fig. 2);

- A work and study space, dedicated for students and researchers;

- Offices, a meeting room and services.

The diversity of the functions present in the laboratory makes it a challenging acoustic environment, as naturally each of these activities requires a specific sonic treatment. The research in this paper focuses on the work and study area, as the one where a lack of proper acoustic conditions could lead to an uncomfortable environment for focused work or even simple conversation or discussion.

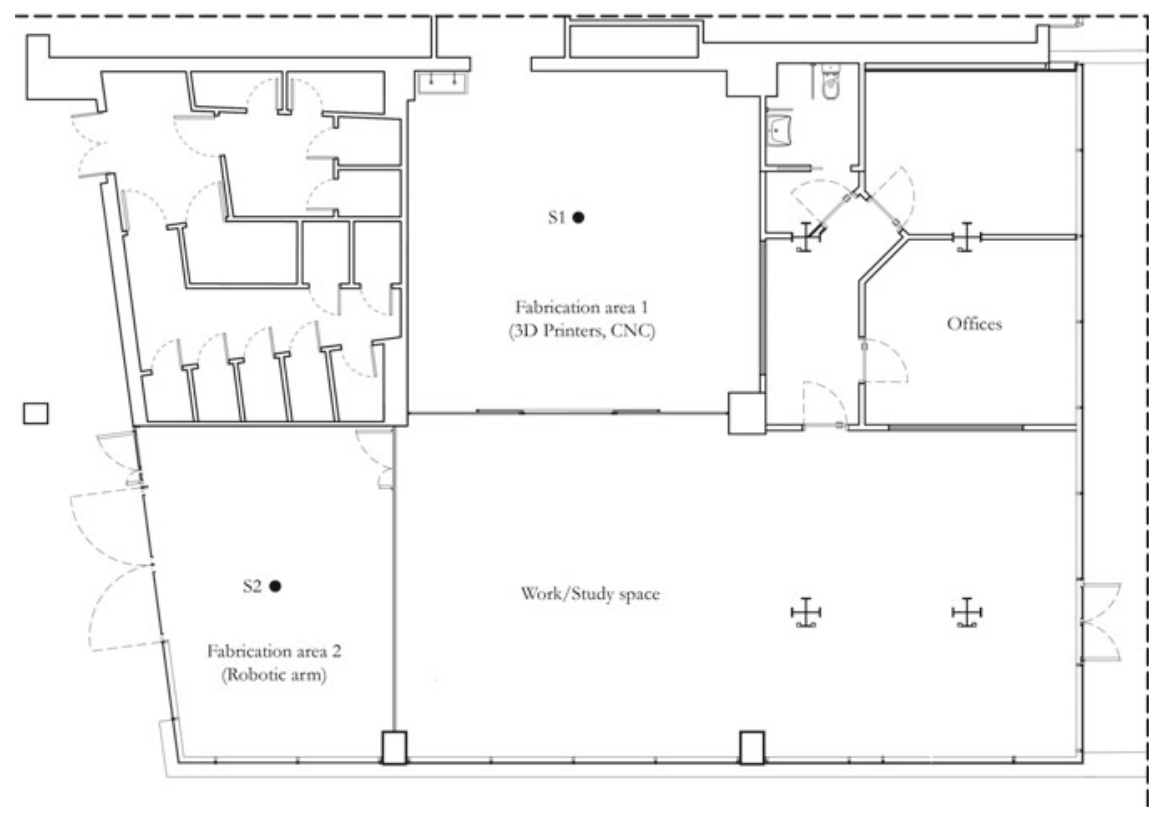

Fig. 2 Plan of the future Digital Fabrication Lab and the main sound sources 


\subsection{Simulation and Analysis of the State-of-the-Art Conditions}

For the acoustic analysis and evaluation of the proposed interior design, simulations were performed with FEA software (Comsol), using the ray acoustics interface in order to understand the high-frequency (reverberant) behaviour of the space. The simulations were made considering the worst conditions, that is, with doors opened towards the work and study area, and with both sound sources active at the same time and employed in full capacity. The maximum sound power level of the robotic arm was hypothesised at $75 \mathrm{~dB}(0.01 \mathrm{~W})$, considering a mill as an end effector, while the value for the sound power level for the CNC and the 3D printers, set as a logarithmic sum, was specified to $105 \mathrm{~dB}(0.03 \mathrm{~W})$.

Two scenarios were considered for the simulations:

- Scenario 0: no acoustic treatment

- Scenario 1: standard acoustical dropped ceiling (200 mm construction depth and $50 \mathrm{~mm}$ mineral wool) (Table 1).

The goal of the acoustic performance analysis was to obtain information about the sound distribution in the space, through reverberation time $\left(\mathrm{T}_{60}\right)$ and time-dependent sound pressure level (SPL), calculated in full octave frequency bands. The results showed that despite the acoustic ceiling in Scenario 1 improves the reverberation time, from 2.75 to $1.2 \mathrm{~s}$, compared to Scenario 0, the SPL levels in the work/study area in the speech-relevant frequency band are still significantly high—average $75 \mathrm{~dB}$ (Fig. 3).

The results were used in the next phase to delineate the areas of intervention, providing the boundary conditions for the spatial distribution of the acoustic surfaces and their geometric characteristics. The objective was to decrease the overall SPL values in the work/study area with at least 10-15 dB (ISO 3382-3), and decrease the reverberation time, employing less surface area than Scenario 1.

Table 1 Absorption coefficients of the materials used for simulation

\begin{tabular}{l|l|l|l|l|l|l}
\hline Materials & $125 \mathrm{~Hz}$ & $250 \mathrm{~Hz}$ & $500 \mathrm{~Hz}$ & $1000 \mathrm{~Hz}$ & $2000 \mathrm{~Hz}$ & $4000 \mathrm{~Hz}$ \\
\hline Wall (plaster) & 0.30 & 0.02 & 0.03 & 0.04 & 0.05 & 0.07 \\
\hline Glass & 0.30 & 0.20 & 0.10 & 0.07 & 0.05 & 0.02 \\
\hline Steel columns & 0.13 & 0.09 & 0.08 & 0.09 & 0.11 & 0.11 \\
\hline Rough concrete & 0.02 & 0.03 & 0.03 & 0.03 & 0.04 & 0.07 \\
\hline Floor (resin) & 0.05 & 0.04 & 0.03 & 0.08 & 0.04 & 0.08 \\
\hline Acoustical ceiling & 0.55 & 0.80 & 0.75 & 0.65 & 0.60 & 0.50 \\
\hline
\end{tabular}



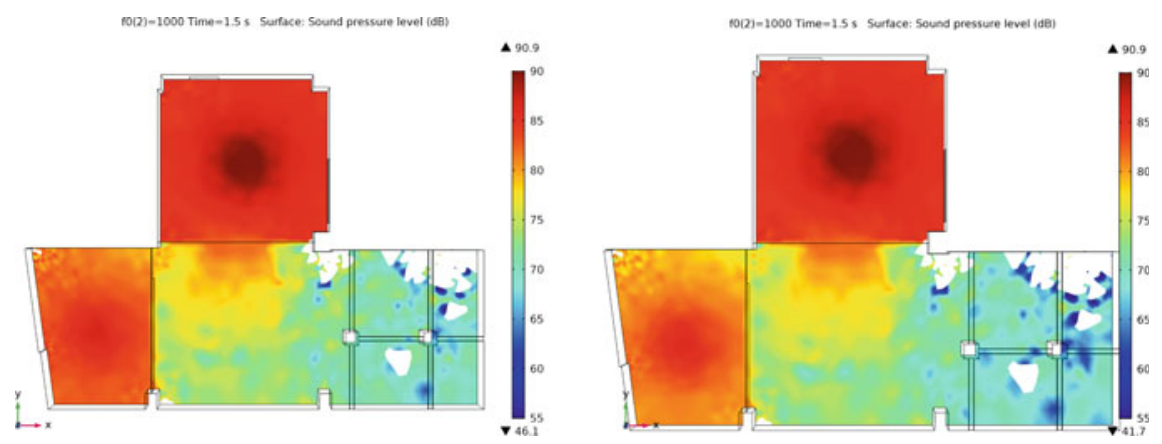

Fig. 3 Sound pressure levels for $1000 \mathrm{~Hz}, 1.5 \mathrm{~s}$ of Scenario 0 (left) and Scenario 1 (right)

\section{Surfaces for Tailored Performance}

Based on the analysis of the state-of-the-art conditions, two acoustic treatment strategies were defined:

- Scenario 2: an optimised solution on the ceiling

- Scenario 3: an optimised self-supporting partition.

Scenario 2 focuses on providing larger area of absorption to lower the reverberation time of the overall space. Scenario 3 instead uses a single partition, aimed at blocking first- and second-order reflections, preventing sound distribution towards the rest of the space. The Rayshoot method (Snail plug-in) was used for the generation of rays from the two sound sources and for calculating the intersection points with the analysed geometries. Multi-objective evolutionary optimisation was performed with the Octopus plug-into generate a diversity of optimised trade-off solutions, ranging between the extremes of each target.

The constraints, used for the geometry generation for Scenario 2 and for determining the boundary conditions of the optimisation process, include setting the displacement of the centre point of the panels in $\mathrm{Z}$ to $1 \mathrm{~m}$, as well as limiting the number of tessellations to four per each face. Owing to the higher sound power level, and the closer proximity to the study area, the absorption from sound source 1 was prioritised (Fig. 4). The optimisation produced a range of candidate solutions, and the one with most optimal values for all the three objectives was selected.

Scenario 3 follows similar form-finding logic and the same objectives of Scenario 2 , but the position of the partition within the space was added as an additional constraint and an opportunity to be moved to match changes in the functional programme (Fig. 5). The boundaries of the possible locations were derived from the SPL maps, resulting from the initial simulation. The selected candidate provides maximum ray intersection values from both the sources. 


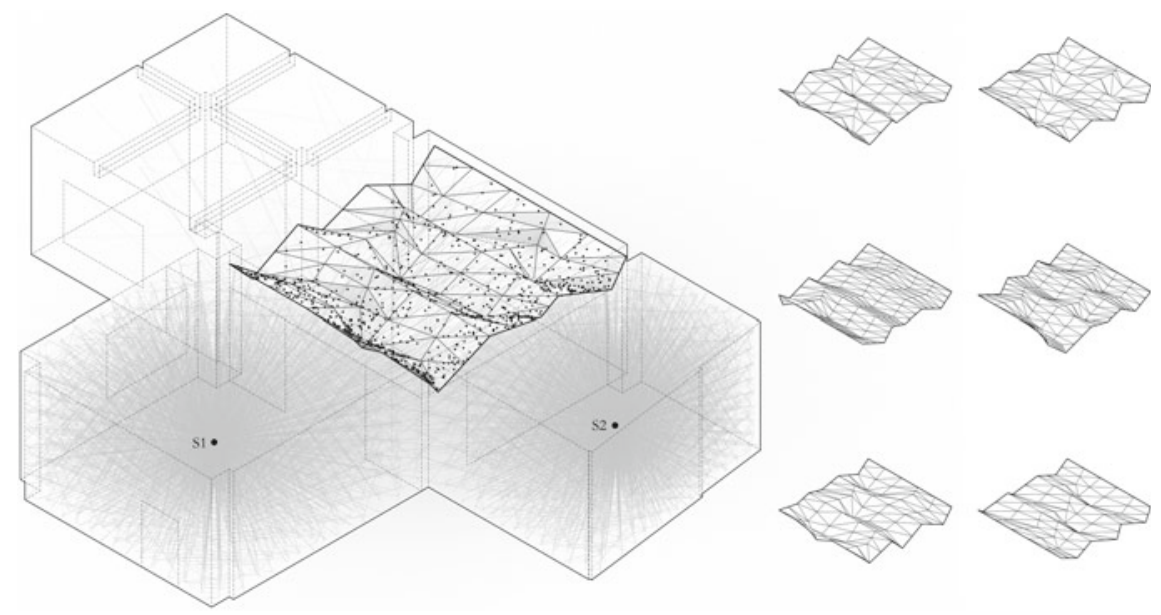

Fig. 4 Form-finding Scenario 2: ceiling (left) and candidate solutions (right)
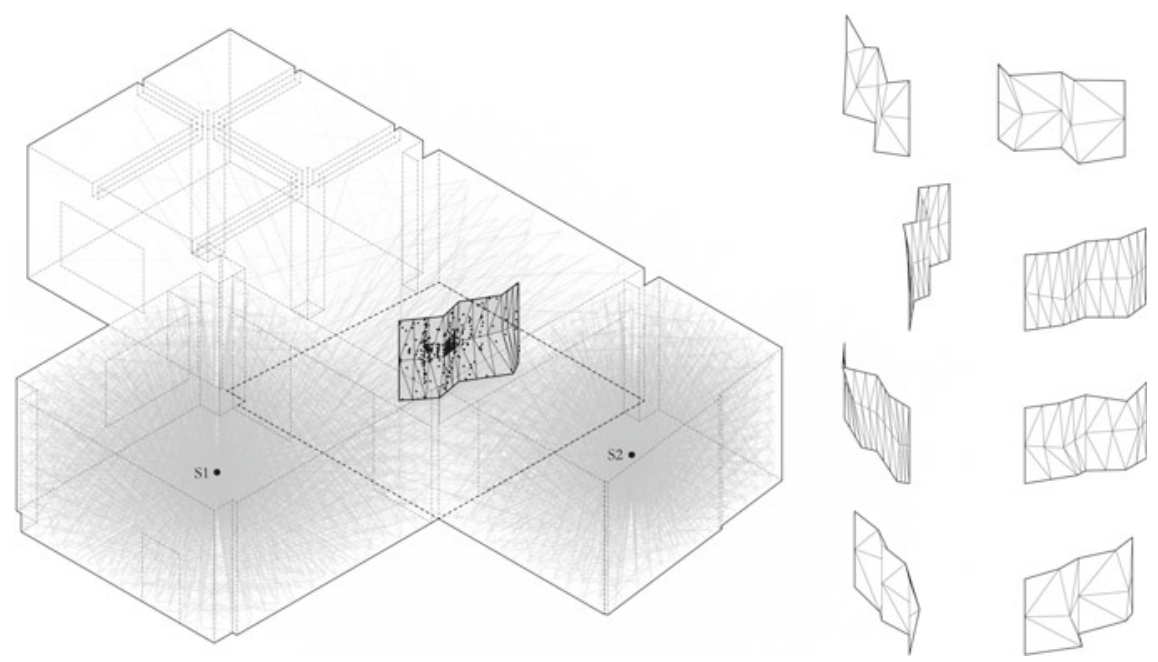

Fig. 5 Form-finding Scenario 2: wall (left) and candidate solutions (right)

\section{Simulation and Results}

The absorption values used for the analysis of the acoustic performance of the selected candidates were taken from the reverberation room tests of the prototypes, developed prior. The a values are: $125 \mathrm{~Hz}-0.55 ; 250 \mathrm{~Hz}-0.80 ; 500 \mathrm{~Hz}-0.75 ; 1000 \mathrm{~Hz}-0.65$; $2000 \mathrm{~Hz}-0.60 ; 4000 \mathrm{~Hz}-0.50$.

The simulation results for Scenario 1 showed that orienting the surfaces provides significant lowering of the overall SPL values within the work/study area, compared 
$f 0(4)=1000$ Time $=1.5 \mathrm{~s}$ Surface: Sound pressure level $(\mathrm{dB})$

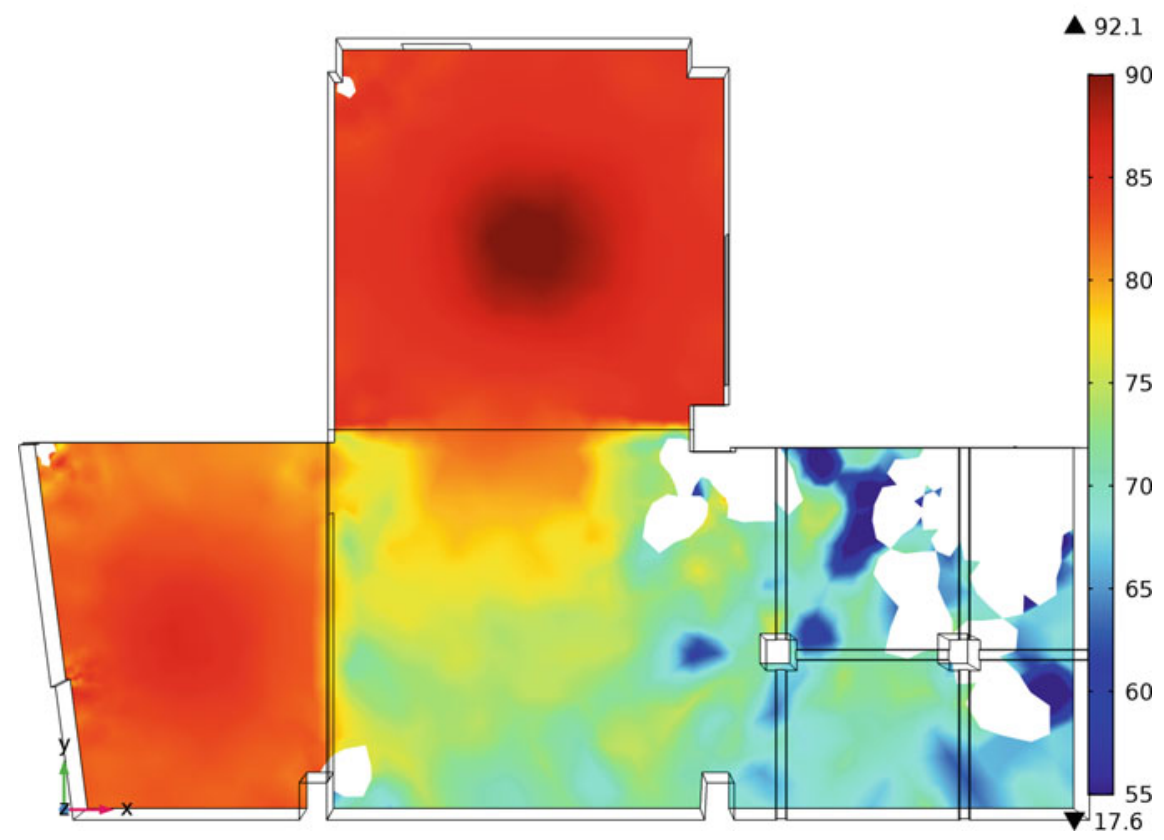

Fig. 6 SPL at $1000 \mathrm{~Hz}, 1.5 \mathrm{~s}$ for Scenario 2: ceiling

to Scenario 1 (Fig. 6). The reverberation time, however, was not affected much, and Scenario 1 provided shorter $\mathrm{T}_{60}$ values. This can be attributed to the fact that the overall surface area of the ceiling in Scenario 2 is much lower.

The results are similar in Scenario 3, showing lower SPL values within the work/study area than Scenario 1 (Fig. 7). This shows that the optimised position of the partition in the space influences early reflections, blocking the sound closer to the source. However, the higher SPL values, compared to Scenario 2, evidence that more absorption surface area is needed for the solution to be fully effective.

\section{Conclusions and Discussion}

The results of the acoustic analysis of the proposed solutions clearly demonstrate that the system, both in ceiling and wall configuration, contributes to creating an improved acoustic environment in the work/study area, compared to standard solutions with dropped acoustic ceiling. The outcomes show that following the proposed methodology for performance-driven form generation in the global scale, it is possible to achieve lower sound pressure levels by means of geometrical optimisation aimed at orienting the absorptive surfaces such as to attenuate sound closer to the 


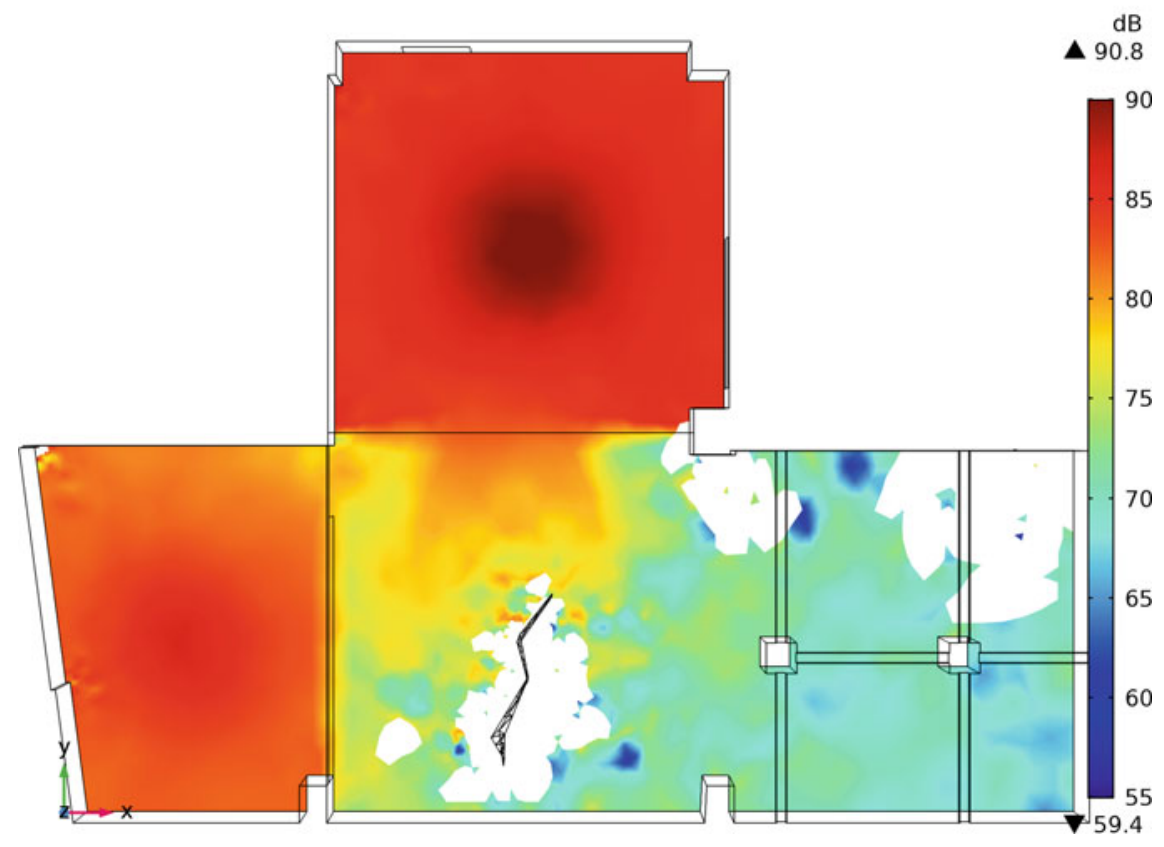

Fig. 7 SPL at $1000 \mathrm{~Hz}, 1.5 \mathrm{~s}$ for Scenario 3: wall

source and impact early reflections. Employing less surface area consequently would also decrease the cost of the overall acoustic treatment.

The innovative aspect of the work is featured in the employment of developable surfaces, which allow the fabrication of geometrically complex panels from a single flat sheet. Moreover, the customised sandwich compositions combined in the WoodSkin technological system offer an additional layer of tailored performance, where the type and thicknesses of the composing materials could be chosen precisely according to the performance targets and functional programme.

The methodology allows the design of acoustic surfaces which not only could be tailored for an optimised absorption but with their inherent corrugations contribute for more uniform sound distribution and increased diffuse sound field, thus limiting the zones with high levels of SPL. The performance criteria will be implemented in the next developments of this project as an additional design target. Future work also includes creation of a custom fabrication process that allows the toolpath optimisation of the openings within the panels, such as the milling time is significantly shortened, leading to lower production cost. Full-scale prototypes will be developed and consequently tested, according to the ISO standards, with the results implemented in a holistic computational process. 


\section{References}

Allen JB, Berkley DA (1979) Image method for efficiently simulating small-room acoustics. J Acoust Soc Am 65(04):943-950. Published by The Acoustical Society of America (ASA)

Bassuet A, Rife D, Dellatorre L (2014) Computational and optimization design in geometric acoustics. Build Acoust 21(1):075-086

Cox TJ, D'Antonio P (2017) Acoustic absorbers and diffusers. In: Theory, design and application. CRC Press

Gladwell GML (1965) A finite element method for acoustics. In: Proceedings of fifth international conference on acoustics

Hargreaves JA, Cox TJ (2010) A transient boundary element method for acoustic scattering from mixed regular and thin rigid bodies. Acta Acust U Acust 95(4):678-689

Jiang J, Huang K, Zhao Y (2011) Calculating room acoustic parameters by finite element method. In: International conference on consumer electronics, communications and networks, CECNet, Proceedings

Kulowski A (1984) Algorithmic representation of the ray tracing technique. Appl Acoust 18:449_ 469

Lee H, Lee B (1988) An efficient algorithm for image method technology. Appl Acoust 24:87-115

Lehnert H (1993) Systematic errors of the ray-tracing algorithm. Appl Acoust 38(2-4):207-221

Lewers T (1993) A combined beam tracing and radiant exchange computer model of room acoustics. Appl Acoust 38(2-4):207-221

Rindel JH (1995) Computer simulation techniques for acoustical design of rooms. Acoust Aust 23(3-81)

Van Maerck D, Martin J (1993) The prediction of echograms and impulse responses within the Epidaure software. Appl Acoust 38(2-4):93

Vorlander M (1989) Simulation of transient and steady state sound propagation in rooms using a new combined ray tracing/image source algorithm

\section{Standards and Laws}

BS EN ISO 354:2003 Acoustics-Measurement of sound absorption in a reverberation room ISO 3382-1 Acoustics-Measurement of room acoustic parameters-Part 1: Performance spaces

Open Access This chapter is licensed under the terms of the Creative Commons Attribution 4.0 International License (http://creativecommons.org/licenses/by/4.0/), which permits use, sharing, adaptation, distribution and reproduction in any medium or format, as long as you give appropriate credit to the original author(s) and the source, provide a link to the Creative Commons license and indicate if changes were made.

The images or other third party material in this chapter are included in the chapter's Creative Commons license, unless indicated otherwise in a credit line to the material. If material is not included in the chapter's Creative Commons license and your intended use is not permitted by statutory regulation or exceeds the permitted use, you will need to obtain permission directly from the copyright holder. 